\title{
ASSOCIATION BETWEEN TOXOPLASMA GONDII TYPES AND OUTCOMES OF HUMAN INFECTION: A META-ANALYSIS
}

\author{
Jing XIA ${ }^{1}$, XIN-Yu Cheng ${ }^{1}$, XiAO-JUn WAnG ${ }^{1,2}$ and Hong-JUAN PENG ${ }^{1 *}$ \\ ${ }^{1}$ Department of Pathogen Biology, Guangdong Provincial Key Laboratory of Tropical \\ Disease Research, and Key Laboratory of Prevention and Control for Emerging Infectious \\ Diseases of Guangdong Higher Institutes, School of Public Health, Southern Medical \\ University, Guangzhou, China \\ ${ }^{2}$ Department of Epidemiology and Biostatistics, School of Public Health, Guangdong \\ Medical University, Dongguan, China
}

(Received: 24 October 2016; accepted: 13 March 2017)

The virulence and pathogenicity of various types of Toxoplasma gondii differ considerably in mice. Recent studies have claimed that similar phenomenon was observed in humans, but no relevant studies have been performed to validate this finding. In addition, reports showing association between a given $T$. gondii type and outcomes of human infection yielded conflicting results. To provide a more precise estimation of the association and a more reliable conclusion on this subject, we performed this meta-analysis. Relevant literatures were identified in multiple databases and selected based on strict screening. T. gondii-type proportions among different severities of infection were calculated and compared using Fisher's exact test. Pooled odds ratios (OR) were calculated. Our results showed that the difference among $T$. gondii-type proportions was significant $(p<0.0001)$. In addition, significant associations were detected between Type I strains infection and congenital toxoplasmosis (OR: $1.91, p=0.0009$ ), Type III strains infection and pulmonary toxoplasmosis (OR: $5.15, p=0.04$ ). In our subgroup analysis, Type I strains were significantly associated with cerebral toxoplasmosis in offspring (OR: 1.81, $p=0.02)$. This result indicated that different types of $T$. gondii exhibited different virulence and caused different outcomes in humans.

Keywords: Toxoplasma gondii, type, human infection, meta-analysis

\section{Introduction}

The obligate intracellular protozoan Toxoplasma gondii is capable of infecting a broad spectrum of warm-blooded vertebrates, including humans [1].

*Corresponding author; E-mail: hongjuan@smu.edu.cn 
T. gondii infection is often acquired through ingestion of water or food that is contaminated by infective oocysts, consumption of meat products containing viable cysts, or vertical transmission of tachyzoites [2]. In humans, the clinical presentations of $T$. gondii infection vary from asymptomatic infection, or non-specific mild symptoms, to severe toxoplasmosis $[3,4]$.

Howe and Sibley [5] originally found a highly clonal population structure of the parasite, which fell into three predominant lineages (Types I, II, and III) by a polymerase chain reaction-restriction fragment length polymorphism (PCRRFLP) analysis, employing $106 \mathrm{~T}$. gondii isolates from Europe and North America. Recently, a PCR-RFLP study employing about 1,500 samples worldwide, revealed 189 different genotypes [6].

The clonal lineages have a number of different phenotypes, such as growth, migration, and transmigration [7]; however, the best characterized of these is their virulence in laboratory mice $[8,9]$. Type I strains exhibit the acute lethal virulence in laboratory mice [lethal dose $\left(\mathrm{LD}_{100}\right) \approx 1$ ], whereas Type II and Type III strains are much less virulent [median lethal dose $\left.\left(\mathrm{LD}_{50}\right) \geq 10^{5}\right][10,11]$.

Several studies suggested that the type of $T$. gondii might be responsible for the variability, or at least part of the variability, on the outcomes of human infection, as it has been observed in laboratory mice $[4,12,13]$. In addition, the growth rate of Type I strains in human foreskin fibroblasts (HFFs) was about one third higher than those of Type II and Type III strains, this fact might reflect that the virulence difference in mice could extend to humans [4]. Accordingly, to provide a more precise estimation of the association between $T$. gondii types and outcomes of human infection, we performed this meta-analysis based on crossregional case-control studies, comprising a total of 1,891 individuals. However, the case-control studies on the strain types not belonging to Types I, II, and III are too limited to generate a meta-analysis, thus we mainly focused on the three clonal lineages and the naturally recombinant strains, which might provide a reference of the virulence in humans for the other types.

\section{Materials and Methods}

\section{Search strategy}

This meta-analysis was performed in accordance with the Cochrane Handbook for Systematic Reviews of Interventions [14] and the Preferred Reporting Items for Systematic Reviews and Meta-Analyses (PRISMA) statement [15] (Supplementary Table I). From multiple databases, including PubMed, Cochrane Library, Web of Science, and China National Knowledge Infrastructure (CNKI) 
database, with the following Medical Subject Heading (MeSH) search terms used individually or in combination with the literature search: "Toxoplasma gondii," "type," "case-control studies," "cohort studies," "human," "asymptomatic," "mild symptoms," and "toxoplasmosis," a total of 1,560 potentially relevant citations were identified. The reference lists of all retrieved articles were also scrutinized for additional relevant studies.

\section{Inclusion and exclusion criteria}

After the exclusion of duplicate articles, the titles and abstracts of the remaining articles were screened by two independent reviewers (JX and XY-C) to identify eligible studies, this screening was followed by an assessment of the full texts based on several criteria for inclusion and exclusion. The inclusion criteria were as follows: (1) the published full text was available; (2) it was an observational study (a case-control study or a cohort study); (3) sufficient data were reported to calculate the odds ratio (OR) or the risk ratio with their $95 \%$ confidence intervals (CIs); and (4) typing of $T$. gondii isolates was based on one of the following genetic or serological methods: (a) PCR-RFLP analysis; (b) microsatellite analysis; (c) multilocus sequence analysis; (d) random amplified polymorphic DNA-PCR; (e) high-resolution melting; (f) ELISA format; (g) peptide-microarray test; or (h) genotype or serotype of $T$. gondii that could be confirmed by medical records. Studies were excluded if they were (1) lacking any raw data or control subjects; (2) included fewer than 10 participants; (3) comments, congresses, abstracts, reviews, or editorials; or (4) reported mix infection (combination of more than one type in one sample).

\section{Data extraction}

The following information were extracted in duplicate (JX and X-YC) from all of the included studies: name of the first author, publication year, country or geographical region, specific descriptions of the infection, genetic markers for genotyping, serological markers for serotyping, reference strain, genotype or serotype, sample size, and the number of the exposure of interest for case-control studies.

\section{Quality assessment}

To assess the methodological quality and risk of bias associated with the included studies, we used the Newcastle-Ottawa Scale (NOS) (Supplementary Table II). The studies that scored more stars were regarded to be of higher quality. 
Studies with four stars for selection, two stars for comparability, and three stars for exposure were considered to have a low risk of bias. Studies having two or three stars for selection, one for comparability, and two for exposure were regarded to have a medium risk of bias. Studies provided with one star for selection or exposure, or no star for any of the three parts, were deemed to have a high risk of bias. Quality assessment and stars allocation were performed by two independent reviewers (JX and X-YC).

\section{Statistical analysis}

Fisher's exact test (Monte Carlo method) was conducted to examine the discrepancy among the $T$. gondii-type proportions of different severities of human infection, and multiple comparisons were performed using the $Z$-test (where $p$ values were adjusted by the Bonferroni method) [16]. Statistical heterogeneity among studies was calculated using the $\chi^{2}$ test, $p$ values, and $I^{2}$ statistics [17]. A random-effects analysis model was used to estimate the overall OR when heterogeneity was significant (Q: $p \leq 0.1$ and $I^{2} \geq 50 \%$ or Q: $p \leq 0.1$ and $I^{2} \geq 25 \%$ ); If the reverse was true, a fixed-effects analysis model was used (Q: $p>0.1$ and $I^{2} \leq 50 \%$ or Q: $p \leq 0.1$ and $I^{2}<25 \%$ ). For the association between $T$. gondii types and outcomes of human infection, the odds of a given $T$. gondii type were compared in the case group (infected population with a given manifestation) versus the control group (infected population without this manifestation). The results were obtained after pooling estimates of each individual study, and reported as the overall OR with a 95\% CI by using Mantel-Haenszel statistical method, and forest plot was also generated. Sensitivity analysis was conducted to examine if the results were robust [18]. Funnel plot was created and its symmetry was checked to evaluate publication bias [19]. Subgroup analysis of the relationship between $T$. gondii types and congenital toxoplasmosis was performed. Fisher's exact test and Z-test were undertaken using IBM SPSS Statistics 20.0 [20]; risk estimates, test of heterogeneity, sensitivity calculation, and publication bias analysis were performed using Review Manager software, version 5.3 [21].

\section{Results}

\section{Definitions}

The term "infection with mild symptoms" used in this study was based on the flu-like and self-limited symptoms of Toxoplasma-infected patients, including fever, swollen lymph nodes, and muscle weakness. It should be noted that if the 
infected patient had "mild symptoms" at the early stage of infection, and developed a severe toxoplasmosis later, then this case was classified as "toxoplasmosis." It was also critical to point out that the strain types not belonging to the three clonal lineages (Types I, II, and III) or recombinant (Types I/II, I/III, and II/III), and the strain types described as "atypical," "exotic," or "unique" directly in the included studies, were defined as "the other types" in this study.

\section{Study characteristics}

Of the 1,560 potentially relevant citations identified, 39 case-control studies published in 15 articles [22-36], comprising a total of 1,891 individuals, met the study criteria and were included in our meta-analysis (Figure 1). The types of T. gondii involved in our analysis were grouped in "clonal lineages" (Types I, II, and III), "recombinant" (Types I/II, I/III, and II/III), and "the other types" (TONT, GPHT, MAS, GANGI, Africa 1, Africa 2, Caribbean 1, Br I, Br II, Br III, ToxoDB\#19, \#108, \#163, \#206, \#226, \#227, \#228, and \#229). Supplementary Table III shows the characteristics of these included studies and their qualities. Figure 2 shows that $1(7 \%)$ [37] of the 15 included studies had a low risk of bias for selection, 13 studies (87\%) [23-30, 32, 34-37] had medium risk, and 1 study (7\%) [22] had a high risk of bias. The risk of bias for comparability was low in 10 studies $(67 \%)[22,24,26,27,29,30,32,34,35,37]$ and medium in 5 studies $(33 \%)[23,25,28,31,36]$. For exposure, 8 studies $(53 \%)[22,23,25,26,30,32$, $34,36]$ had a low risk of bias and 7 studies $(47 \%)[24,27-29,31,35,37]$ had a high risk of bias.

\section{Analysis of contingency table}

Fisher's exact test. Discrepancy among the strain types' proportions of the three severities of human infection (asymptomatic infection, mild symptoms, and toxoplasmosis) was examined with Fisher's exact test (Monte Carlo method) since more than $20 \%$ of the cells in the contingency table had expected counts that were less than 5 . The types' distributions of the three human severities are shown in Figure 3 and Supplementary Table IV. The result of Fisher's exact test revealed a significant difference in types' proportions among the three severities (value $=254.375, p<0.0001$, see Supplementary Table V).

Multiple comparisons. As the significant discrepancy was determined, multiple comparisons of the proportions among the three severities for a given type were subsequently performed using the $Z$-test. According to the result of multiple comparisons (Table I), the proportions of patients with mild symptoms (13.9\%) 


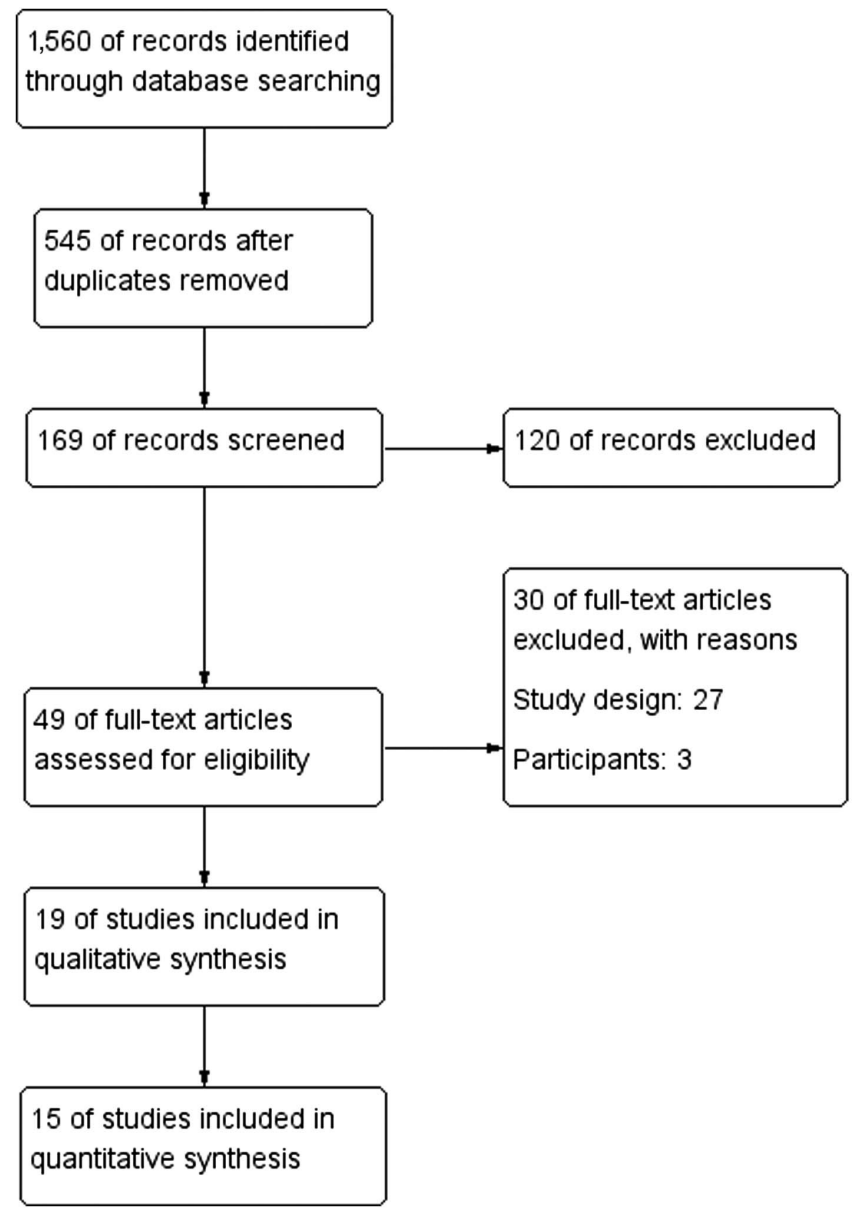

Figure 1. Flowchart of study selection

and toxoplasmosis $(17.7 \%)$ were significantly higher than those with no symptom (2.7\%) for Type I strains infection. For Type II strains infection, a significant low proportion was found in patients with mild symptoms (26.6\%) compared with those with no symptoms (51.7\%) and toxoplasmosis (59.7\%). Interestingly, as with Type I strains infection, the Type III strains infection proportions of patients with mild symptoms $(8.9 \%)$ and toxoplasmosis $(8.6 \%)$ were significantly higher than those with no symptoms (1.1\%). For recombinant Types I/II and II/III, a significant higher proportion was found in patients with mild symptoms $(3.8 \%$ and $24.1 \%$, respectively), whereas for recombinant Type I/III, the proportion of infected population without any symptoms $(43.0 \%)$ was significantly higher than 


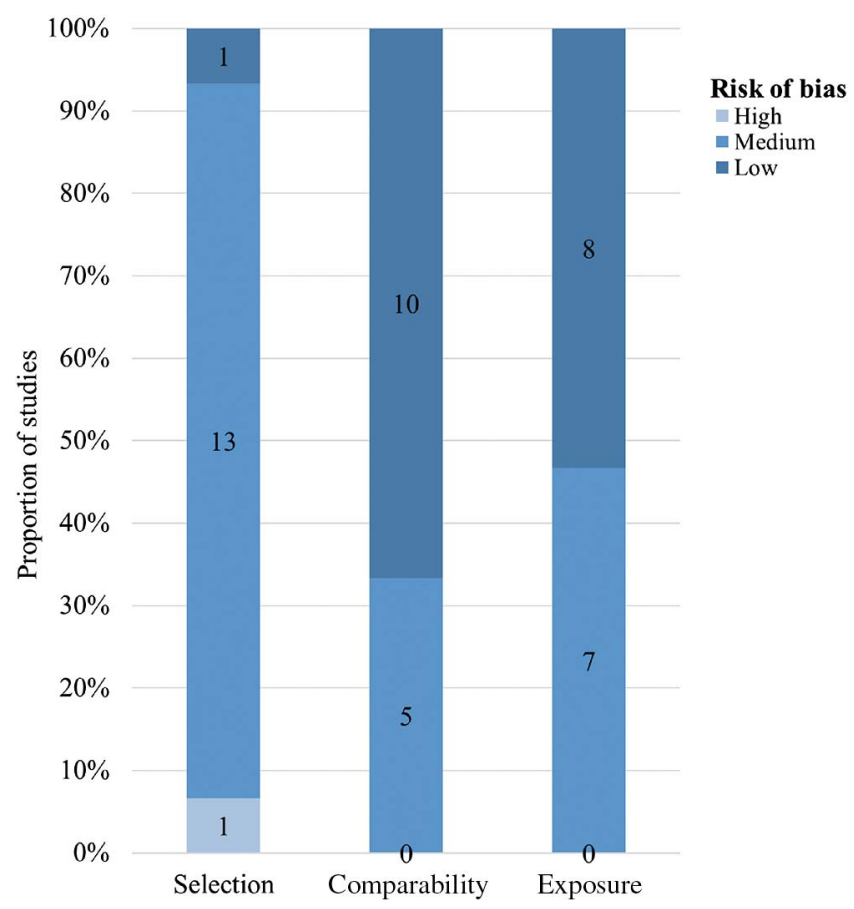

Figure 2. Quality assessment using the NOS for risk of bias of included studies. The absolute number of studies was shown in boxes. For selection, $1(7 \%)$ of the 15 included studies had low risk of bias, $13(87 \%)$ had medium risk, and 1 (7\%) had high risk of bias; for comparability, $10(67 \%)$ studies had low risk and $5(33 \%)$ studies had medium risk; and for health outcomes, $8(53 \%)$ studies had low risk and $7(47 \%)$ studies had medium risk of bias

the other two severities. For the other types, no significant difference was found among the three severities.

\section{Meta-analysis}

T. gondii types and asymptomatic infection. In total, five case-control studies [23, $24,27,28,36]$ were included to perform the meta-analyses based on the association between $T$. gondii types and asymptomatic infection. However, our results did not reveal a significant association between $T$. gondii types and asymptomatic infection in humans $(p>0.05)$ (Supplementary Table IX).

T. gondii types and infection with mild symptoms. For $T$. gondii types and infection with mild symptoms, the overall OR with its $95 \%$ CI was extracted from 


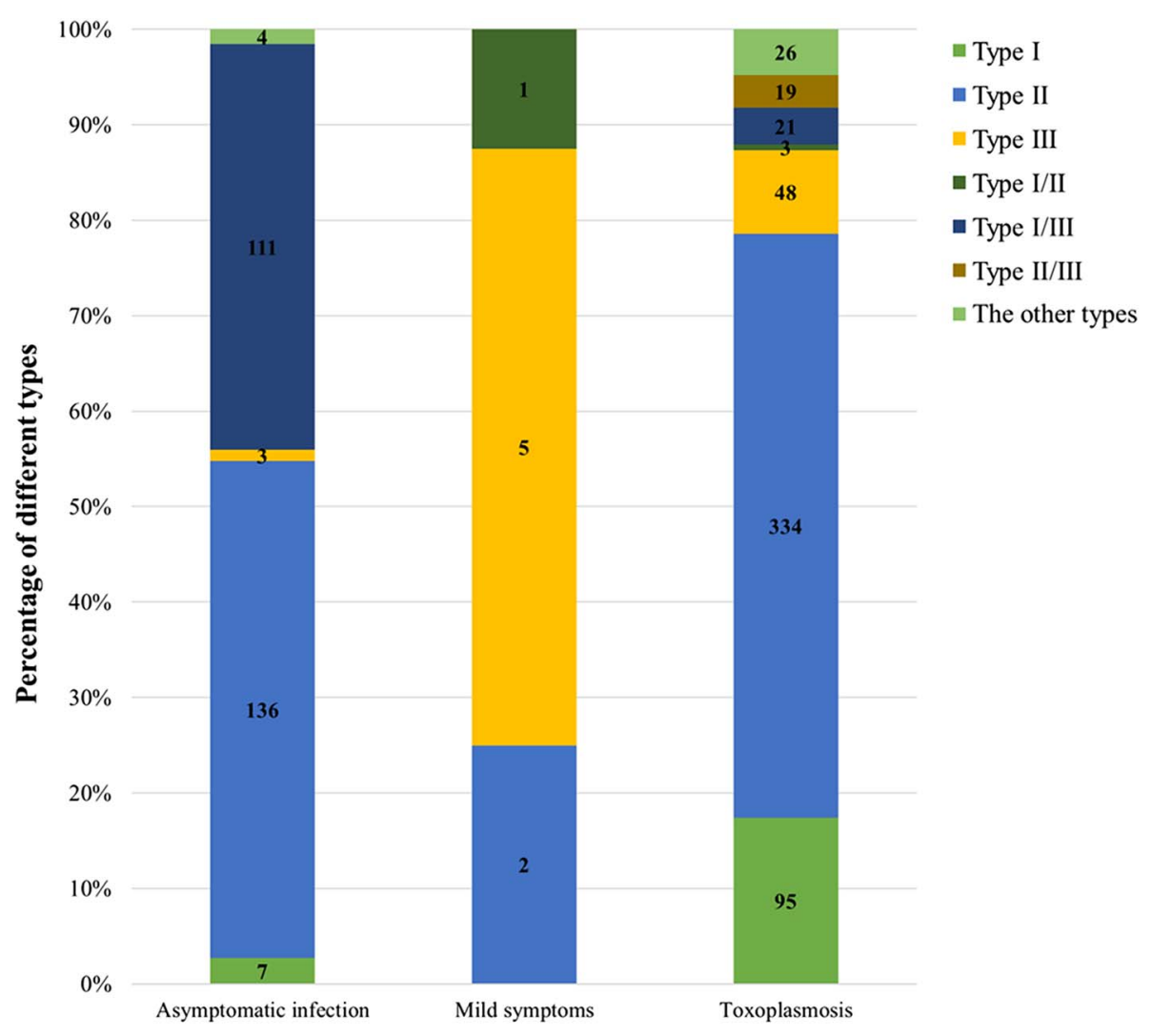

Severity of Toxoplasma gondii infection

Figure 3. Distribution of $T$. gondii types by severity of human infection. Different patterns of types' distribution of the three severities demonstrated that different types of $T$. gondii could cause illness in humans at different severities. The highest proportions of Type II strains presented in the groups of asymptomatic infection and toxoplasmosis indicated a strong association between Type II strains and human infection

two included case-control studies [25, 29]. No significant difference was reported between the case groups and control groups for Type II strains exposure (OR: 0.98, 95\% CI: 0.16-6.19, $p=0.98$ ) (Supplementary Table IX).

T. gondii types and human toxoplasmosis. In the meta-analysis of $T$. gondii types and risk of human toxoplasmosis, 26 case-control studies published in 15 articles were included [22-32, 34-37] (Supplementary Tables VI-VIII). The result showed that the odds of Type I strains infection were significantly higher in patients with congenital toxoplasmosis compared with those without congenital 
Table I. Results of multiple comparison analysis

\begin{tabular}{|c|c|c|c|c|c|c|}
\hline & & & \multicolumn{3}{|c|}{ Severities } & \multirow[b]{2}{*}{ Total } \\
\hline & & & $\begin{array}{c}\text { Asymptomatic } \\
\text { infection }\end{array}$ & $\begin{array}{c}\text { Mild } \\
\text { symptoms }\end{array}$ & Toxoplasmosis & \\
\hline \multirow[t]{14}{*}{ Types } & I & Count & $7 \mathrm{a}$ & $0_{\mathrm{a}, \mathrm{b}}$ & $95_{b}$ & 102 \\
\hline & & $\%$ within severities & 2.7 & 0.0 & 17.4 & 12.5 \\
\hline & II & Count & $136_{\mathrm{a}}$ & $2 \mathrm{a}, \mathrm{b}$ & $334_{b}$ & 472 \\
\hline & & $\%$ within severities & 52.1 & 25.0 & 61.2 & 57.9 \\
\hline & III & Count & $3 \mathrm{a}$ & $5_{\mathrm{b}}$ & $48_{\mathrm{c}}$ & 56 \\
\hline & & $\%$ within severities & 1.1 & 62.5 & 8.8 & 6.9 \\
\hline & $\mathrm{I} / \mathrm{II}$ & Count & $0_{\mathrm{a}}$ & $1_{\mathrm{b}}$ & $3_{\mathrm{a}}$ & 4 \\
\hline & & $\%$ within severities & 0.0 & 12.5 & 0.5 & 0.5 \\
\hline & I/III & Count & $111_{\mathrm{a}}$ & $0_{\mathrm{b}}$ & $21_{\mathrm{b}}$ & 132 \\
\hline & & $\%$ within severities & 42.5 & 0.0 & 3.8 & 16.2 \\
\hline & II/III & Count & $0_{\mathrm{a}}$ & $0_{\mathrm{a}, \mathrm{b}}$ & $19_{\mathrm{b}}$ & 19 \\
\hline & & $\%$ within severities & 0.0 & 0.0 & 3.5 & 2.3 \\
\hline & $\begin{array}{c}\text { The other } \\
\text { types }\end{array}$ & Count & $4 a$ & $0_{\mathrm{a}}$ & $26_{a}$ & 30 \\
\hline & & $\%$ within severities & 1.5 & 0.0 & 4.8 & 3.7 \\
\hline \multirow[t]{2}{*}{ Total } & & Count & 261 & 8 & 546 & 815 \\
\hline & & $\%$ within severities & 100.0 & 100.0 & 100.0 & 100.0 \\
\hline
\end{tabular}

Note: Each subscript letter denotes a subset of severities categories whose column proportions do not differ significantly from each other at the 0.05 level.

toxoplasmosis (OR: 1.91, 95\% CI: 1.31-2.80, $p=0.0009$ ) (Table II and Supplementary Table VI). In addition, the result showed a significantly heightened odds of Type III strains infection in patients with pulmonary toxoplasmosis versus those without pulmonary toxoplasmosis (OR: 5.15, 95\% CI: $1.05-25.26, p=0.04$ ) (Table II and Supplementary Table VII).

Subgroup analysis. Subgroup analysis of the relationship between the $T$. gondii types and congenital toxoplasmosis was performed based on the following pathological changes: maternal seroconversion from negative to positive; abortion, fetal death, or newborn death; cerebral toxoplasmosis in newborns or in later life; and ocular toxoplasmosis in newborns. All subgroup differences were small (tests for subgroup differences: $p>0.1$ ) (Supplementary Table X). Significant positive result was obtained in the associations of Type I strains infection with cerebral toxoplasmosis in offspring (fetus, newborns, or children) (OR: 1.81, 95\% CI: 1.11-2.97, $p=0.02$ ) (Table II and Supplementary Table VIII).

Sensitivity analysis. To identify whether the result of the meta-analysis was significantly affected by exclusion of the study with the highest quality or 
Table II. Results of meta-analysis on relationship between $T$. gondii type and toxoplasmosis

\begin{tabular}{|c|c|c|c|c|c|}
\hline \multirow[b]{2}{*}{ Clinical presentations } & \multirow[b]{2}{*}{ Total } & \multicolumn{2}{|c|}{ Toxoplasmosis } & \multirow[b]{2}{*}{ OR $(95 \% \mathrm{CI})$} & \multirow[b]{2}{*}{$p$ value } \\
\hline & & Number & Percent & & \\
\hline \multicolumn{6}{|c|}{ Congenital toxoplasmosis } \\
\hline \multicolumn{6}{|c|}{$T$. gondii type I } \\
\hline Case & 327 & 64 & 19.6 & $1.91(1.31,2.80)$ & 0.0009 \\
\hline Control & 1,076 & 105 & 9.8 & & \\
\hline \multicolumn{6}{|c|}{ Pulmonary toxoplasmosis } \\
\hline \multicolumn{6}{|c|}{ T. gondii type III } \\
\hline Case & 10 & 3 & 30.0 & $5.15(1.05,25.26)$ & 0.04 \\
\hline Control & 90 & 8 & 8.9 & & \\
\hline \multicolumn{6}{|c|}{ Cerebral toxoplasmosis in offsprings } \\
\hline \multicolumn{6}{|c|}{ T. gondii type I } \\
\hline Case & 221 & 28 & 12.7 & $1.81(1.11,2.97)$ & 0.02 \\
\hline Control & 638 & 53 & 8.3 & & \\
\hline
\end{tabular}

the study with the greatest weight in results, sensitivity analyses were conducted. No significant impact was observed in the overall ORs and 95\% CIs.

Publication bias. Publication bias of the studies included in this meta-analysis was examined using funnel plots (Figure 4). All the plots in the four outcomes approximately resembled a symmetrical funnel, hence no publication bias was found.

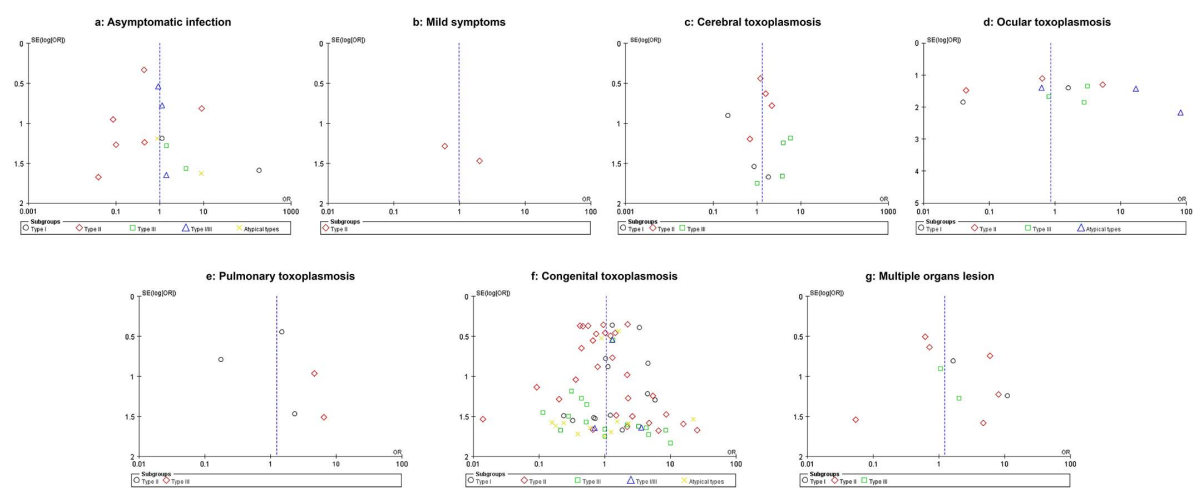

Figure 4. Funnel plots of studies on human infection with T. gondii. (a) Funnel plot of studies on asymptomatic infection; (b) Funnel plot of studies on infection with mild symptoms; (c-g) Funnel plots of studies on toxoplasmosis. The plots approximately resembled a symmetrical funnel, indicating the absence of publication bias 


\section{Discussion}

It has long been known that the Type I strains of $T$. gondii are highly virulent, whereas Type II or Type III strains are less virulent or non-virulent in mice $[10,11]$. Because of the broad range of hosts that the parasite can infect, it is unclear whether the virulence difference observed in mice will also be observed in other hosts, such as humans. In this study, we compared the strain proportions among the three severities of human infection, and tested if the proportions of one given $T$. gondii type were significantly different among the three severities, which would provide an indication of the characteristics of $T$. gondii virulence in humans. According to the result of Fisher's exact test, the difference among the three proportions was significant (value $=254.375, p<0.0001$ ), suggesting different types of $T$. gondii could cause illness in humans at different severity levels, indicating different types of $T$. gondii might present different virulence in humans. Various polymorphic effectors secreted from rhoptry and dense granule contributed to the different virulence expression in different types of $T$. gondii [38-40], including ROP18, ROP16, and GRA15 [41-43]. These findings were not only observed in the mouse model, but also in HFFs, which gave a hint that the polymorphism of these effectors might be responsible for different virulence presented in humans, even though little was known regarding $T$. gondii virulence effectors in human infection.

We also found that there was an extremely strong association between Type II strains and human infection because the highest proportions of Type II strains were presented in groups of asymptomatic infection and toxoplasmosis (Figure 3). Several studies have also indicated this similar association. In North America, Type II strains were most commonly associated with human infection, including in patients with congenital toxoplasmosis and patients with AIDS $[5,22]$. In Europe, the same trend that Type II strains predominate in human infection was observed [24, 44]. Such common exposure to this strain type required our attention and this striking association was important for prevention, diagnosis, and treatment of human infection.

Our analysis of the multiple comparison of Type I strains infection revealed significant higher proportions of patients with toxoplasmosis and mild symptoms than those with asymptomatic infection, which suggested that Type I strains mainly caused apparent diseases or mild symptoms, potentially with a medium to high level of virulence in humans. Previously, a cross-sectional study reported a predominance of Type I parasites infection in ocular toxoplasmosis patients [45], whereas in another study, Type I strains were reported to be more frequently associated with congenital toxoplasmosis in humans [46]. According to our 
meta-analysis, a significant association was observed between Type I T. gondii infection and congenital toxoplasmosis (OR: 1.91, $p=0.0009$ ). It is reported that the Type I strains could generate significantly higher levels of parasitemia, and increase both the risk of transplacental transmission and the severity of infection in fetus or newborn [5, 47]. Our subgroup analysis confirmed the significant association of Type I strains infection with cerebral toxoplasmosis in offspring (fetus, newborns, or children) (OR: 1.81, $p=0.02$ ). The mechanism for this was unknown, but it was possible that during pregnancy, the acute infection acquired from the Type I parasites could cause higher parasitemia and an increased rate of congenital infection $[5,31]$, since the parasite was apt to infect the nervous system, therefore, the Type I strains were predominant in congenital toxoplasmosis [31]. In addition, the mother's anti-toxoplasma antibodies might influence the prenatal or postnatal ontogeny of a child [48].

To our surprise, the Type III strains infection proportions of patients with mild symptoms and toxoplasmosis were significantly higher than those with asymptomatic infection, similar to the Type I strains infection. One difference, however, was that the proportion of patients with mild symptoms was a little higher than those with toxoplasmosis for Type III infection, while the reverse result was observed for Type I infection. These results indicated that Type III strains of $T$. gondii might present medium virulence in humans, unlike their avirulence in laboratory mice. In our meta-analysis, the significant association between the Type III strains infection and pulmonary toxoplasmosis was confirmed (OR: 5.15, 95\% CI: 1.05-25.26, $p=0.04$ ). The pathomechanism of Type III parasites in pulmonary toxoplasmosis was unknown, but it was reported that Type III parasites infection could result in change of a critical enzyme TDO2, in the kynurenine pathway in infected cells [33], this finding implicated a complex pathogenesis of Type III $T$. gondii.

In this study, we revealed an apparent difference of $T$. gondii virulence between laboratory mice and humans, at least for the three clonal lineages and their recombinant strains. It was possible that there was difference in susceptibility to $T$. gondii, or to different types of the parasite, between mice and humans. Alternatively, it was also possible that there was difference in pathogenic mechanism of the parasite during infection between mice and humans. However, it was notable that in addition to the parasite types, other factors, such as the genetic backgrounds of hosts, immunity condition of hosts, and the timing of primary infection might also play a role in outcomes of human infection. In addition, since human isolates were mainly collected from symptomatic cases, the information of $T$. gondii types from asymptomatic cases was always neglected and limited. Including a larger number of studies and studies with larger sample sizes would be necessary to confirm and extend the findings in this study. 


\section{Conclusions}

In conclusion, the meta-analysis suggested different clonal lineages of T. gondii might exhibit different virulence and caused different outcomes in humans. Type I strains infection was significantly associated with an increased risk of human congenital toxoplasmosis, especially the cerebral toxoplasmosis in offspring. Type III parasites showed a significant association with human pulmonary toxoplasmosis.

\section{Acknowledgements}

The authors are grateful to Qiang Huang for statistical advice and Hai-Xia Wei for providing additional information on their request. The authors are much indebted to the funding of the National Natural Science Foundation of China (Nos. 81271866 and 81572012), the Guangdong Province Universities and Colleges Pearl River Scholar Funded Scheme (2014), the Guangdong Provincial Natural Science Foundation Key Project (2016A030311025), and Guangzhou Health and Medical Collaborative Innovation Major Special Project (201604020011) to H-JP for providing the financial support.

JX: study designing, data collection, software operation, data analysis, and drafting of the manuscript. X-YC: data collection and manuscript revision. X-JW: software operation and data analysis. H-JP: study designing, manuscript revision, and manuscript submission. All of the authors read and approved the final version of the manuscript.

\section{Conflict of Interest}

The authors declare no conflict of interest.

\section{References}

1. Blader, I. J., Saeij, J. P.: Communication between Toxoplasma gondii and its host: Impact on parasite growth, development, immune evasion, and virulence. APMIS 117, 458-476 (2009).

2. Tenter, A. M., Heckeroth, A. R., Weiss, L. M.: Toxoplasma gondii: From animals to humans. Int J Parasitol 30, 1217-1258 (2000).

3. Montoya, J. G., Liesenfeld, O.: Toxoplasmosis. Lancet 363, 1965-1976 (2004).

4. Boothroyd, J. C., Grigg, M. E.: Population biology of Toxoplasma gondii and its relevance to human infection: Do different strains cause different disease? Curr Opin Microbiol $\mathbf{5}$, 438-442 (2002). 
5. Howe, D. K., Sibley, L. D.: Toxoplasma gondii comprises three clonal lineages: Correlation of parasite genotype with human disease. J Infect Dis 172, 1561-1566 (1995).

6. Shwab, E. K., Zhu, X. Q., Majumdar, D., Pena, H. F., Gennari, S. M., Dubey, J. P., Su, C.: Geographical patterns of Toxoplasma gondii genetic diversity revealed by multilocus PCRRFLP genotyping. Parasitology 141, 453-461 (2014).

7. Barragan, A., Sibley, L. D.: Migration of Toxoplasma gondii across biological barriers. Trends Microbiol 11, 426-430 (2003).

8. Howe, D. K., Summers, B. C., Sibley, L. D.: Acute virulence in mice is associated with markers on chromosome VIII in Toxoplasma gondii. Infect Immun 64, 5193-5198 (1996).

9. Mordue, D. G., Monroy, F., La Regina, M., Dinarello, C. A., Sibley, L. D.: Acute toxoplasmosis leads to lethal overproduction of Th1 cytokines. J Immunol 167, 45744584 (2001).

10. Sibley, L. D., Ajioka, J. W.: Population structure of Toxoplasma gondii: Clonal expansion driven by infrequent recombination and selective sweeps. Annu Rev Microbiol 62, 329351 (2008).

11. Sibley, L. D., Boothroyd, J. C.: Virulent strains of Toxoplasma gondii comprise a single clonal lineage. Nature 359, 82-85 (1992).

12. Xiao, J., Yolken, R. H.: Strain hypothesis of Toxoplasma gondii infection on the outcome of human diseases. Acta Physiol (Oxf) 213, 828-845 (2015).

13. Sibley, L. D., Khan, A., Ajioka, J. W., Rosenthal, B. M.: Genetic diversity of Toxoplasma gondii in animals and humans. Philos Trans R Soc Lond B Biol Sci 364, 2749-2761 (2009).

14. Higgins, J., Green, S.: Cochrane Handbook for Systematic Reviews of Interventions Version 5.1.0 [updated March 2011]. The Cochrane Collaboration, London, UK, 2011.

15. Moher, D., Liberati, A., Tetzlaff, J., Altman, D. G.: Preferred reporting items for systematic reviews and meta-analyses: The PRISMA statement. Int J Surg 8, 336-341 (2010).

16. Mehta, C. R.: The exact analysis of contingency tables in medical research. Stat Methods Med Res 3, 135-156 (1994).

17. Higgins, J. P., Thompson, S. G.: Quantifying heterogeneity in a meta-analysis. Stat Med 21, 1539-1558 (2002).

18. Stroup, D. F., Berlin, J. A., Morton, S. C., Olkin, I., Williamson, G. D., Rennie, D., Moher, D., Becker, B. J., Sipe, T. A., Thacker, S. B.: Meta-analysis of observational studies in epidemiology: A proposal for reporting. Meta-analysis Of Observational Studies in Epidemiology (MOOSE) group. JAMA 283, 2008-2012 (2000).

19. Sterne, J. A., Egger, M.: Funnel plots for detecting bias in meta-analysis: Guidelines on choice of axis. J Clin Epidemiol 54, 1046-1055 (2001).

20. Takahashi, Y.: Co-immunoprecipitation from transfected cells. Methods Mol Biol 1278, 381-389 (2015).

21. Review Manager (RevMan). Version 5.3. The Nordic Cochrane Centre, The Cochrane Collaboration, Copenhagen, Denmark, 2014.

22. Howe, D. K., Honore, S., Derouin, F., Sibley, L. D.: Determination of genotypes of Toxoplasma gondii strains isolated from patients with toxoplasmosis. J Clin Microbiol 35, 1411-1414 (1997).

23. Fuentes, I., Rubio, J. M., Ramirez, C., Alvar, J.: Genotypic characterization of Toxoplasma gondii strains associated with human toxoplasmosis in Spain: Direct analysis from clinical samples. J Clin Microbiol 39, 1566-1570 (2001). 
24. Ajzenberg, D., Cogne, N., Paris, L., Bessieres, M. H., Thulliez, P., Filisetti, D., Pelloux, H., Marty, P., Darde, M. L.: Genotype of 86 Toxoplasma gondii isolates associated with human congenital toxoplasmosis, and correlation with clinical findings. J Infect Dis 186, 684-689 (2002).

25. Aspinall, T. V., Guy, E. C., Roberts, K. E., Joynson, D. H., Hyde, J. E., Sims, P. F.: Molecular evidence for multiple Toxoplasma gondii infections in individual patients in England and Wales: Public health implications. Int J Parasitol 33, 97-103 (2003).

26. Gallego, C., Saavedra-Matiz, C., Gomez-Marin, J. E.: Direct genotyping of animal and human isolates of Toxoplasma gondii from Colombia (South America). Acta Trop 97, 161-167 (2006).

27. Nowakowska, D., Colon, I., Remington, J. S., Grigg, M., Golab, E., Wilczynski, J., Sibley, L. D.: Genotyping of Toxoplasma gondii by multiplex PCR and peptide-based serological testing of samples from infants in Poland diagnosed with congenital toxoplasmosis. J Clin Microbiol 44, 1382-1389 (2006).

28. Sousa, S., Ajzenberg, D., Vilanova, M., Costa, J., Darde, M. L.: Use of GRA6-derived synthetic polymorphic peptides in an immunoenzymatic assay to serotype Toxoplasma gondii in human serum samples collected from three continents. Clin Vaccine Immunol 15, 1380-1386 (2008).

29. Messaritakis, I., Detsika, M., Koliou, M., Sifakis, S., Antoniou, M.: Prevalent genotypes of Toxoplasma gondii in pregnant women and patients from Crete and Cyprus. Am J Trop Med Hyg 79, 205-209 (2008).

30. Ajzenberg, D., Yera, H., Marty, P., Paris, L., Dalle, F., Menotti, J., Aubert, D., Franck, J., Bessieres, M. H., Quinio, D., Pelloux, H., Delhaes, L., Desbois, N., Thulliez, P., Robert-Gangneux, F., Kauffmann-Lacroix, C., Pujol, S., Rabodonirina, M., Bougnoux, M. E., Cuisenier, B., Duhamel, C., Duong, T. H., Filisetti, D., Flori, P., Gay-Andrieu, F., Pratlong, F., Nevez, G., Totet, A., Carme, B., Bonnabau, H., Darde, M. L., Villena, I.: Genotype of 88 Toxoplasma gondii isolates associated with toxoplasmosis in immunocompromised patients and correlation with clinical findings. J Infect Dis 199, 1155-1167 (2009).

31. Xiao, J., Buka, S. L., Cannon, T. D., Suzuki, Y., Viscidi, R. P., Torrey, E. F., Yolken, R. H.: Serological pattern consistent with infection with type I Toxoplasma gondii in mothers and risk of psychosis among adult offspring. Microbes Infect 11, 1011-1018 (2009).

32. McLeod, R., Boyer, K. M., Lee, D., Mui, E., Wroblewski, K., Karrison, T., Noble, A. G., Withers, S., Swisher, C. N., Heydemann, P. T., Sautter, M., Babiarz, J., Rabiah, P., Meier, P., Grigg, M. E.: Prematurity and severity are associated with Toxoplasma gondii alleles (NCCCTS, 1981-2009). Clin Infect Dis 54, 1595-1605 (2012).

33. Xiao, J., Li, Y., Jones-Brando, L., Yolken, R. H.: Abnormalities of neurotransmitter and neuropeptide systems in human neuroepithelioma cells infected by three Toxoplasma strains. J Neural Transm 120, 1631-1639 (2013).

34. Silva, L. A., Andrade, R. O., Carneiro, A. C., Vitor, R. W.: Overlapping Toxoplasma gondii genotypes circulating in domestic animals and humans in Southeastern Brazil. PLoS One 9, e90237 (2014).

35. Herrmann, D. C., Maksimov, P., Hotop, A., Gross, U., Daubener, W., Liesenfeld, O., Pleyer, U., Conraths, F. J., Schares, G.: Genotyping of samples from German patients with ocular, cerebral and systemic toxoplasmosis reveals a predominance of Toxoplasma gondii type II. Int J Med Microbiol 304, 911-916 (2014). 
36. Tolba, M. M., El-Taweel, H. A., Khalil, S. S., Hazzah, W. A., Heshmat, M. G.: Genotype analysis of $T$. gondii strains associated with human infection in Egypt. Parasitol Res 113, 1563-1569 (2014).

37. Xiao, J., Viscidi, R. P., Kannan, G., Pletnikov, M. V., Li, Y., Severance, E. G., Yolken, R. H., Delhaes, L.: The Toxoplasma MAG1 peptides induce sex-based humoral immune response in mice and distinguish active from chronic human infection. Microbes Infect 15, 74-83 (2013).

38. Saeij, J. P., Boyle, J. P., Coller, S., Taylor, S., Sibley, L. D., Brooke-Powell, E. T., Ajioka, J. W., Boothroyd, J. C.: Polymorphic secreted kinases are key virulence factors in toxoplasmosis. Science 314, 1780-1783 (2006).

39. Taylor, S., Barragan, A., Su, C., Fux, B., Fentress, S. J., Tang, K., Beatty, W. L., Hajj, H. E., Jerome, M., Behnke, M. S., White, M., Wootton, J. C., Sibley, L. D.: A secreted serinethreonine kinase determines virulence in the eukaryotic pathogen Toxoplasma gondii. Science 314, 1776-1780 (2006).

40. Rosowski, E. E., Lu, D., Julien, L., Rodda, L., Gaiser, R. A., Jensen, K. D., Saeij, J. P.: Strain-specific activation of the NF-kB pathway by GRA15, a novel Toxoplasma gondii dense granule protein. J Exp Med 208, 195-212 (2011).

41. Yamamoto, M., Standley, D. M., Takashima, S., Saiga, H., Okuyama, M., Kayama, H., Kubo, E., Ito, H., Takaura, M., Matsuda, T., Soldati-Favre, D., Takeda, K.: A single polymorphic amino acid on Toxoplasma gondii kinase ROP16 determines the direct and strain-specific activation of Stat3. J Exp Med 206, 2747-2760 (2009).

42. Ong, Y. C., Reese, M. L., Boothroyd, J. C.: Toxoplasma rhoptry protein 16 (ROP16) subverts host function by direct tyrosine phosphorylation of STAT6. J Biol Chem $\mathbf{2 8 5}$, 28731-28740 (2010).

43. Yamamoto, M., Ma, J. S., Mueller, C., Kamiyama, N., Saiga, H., Kubo, E., Kimura, T., Okamoto, T., Okuyama, M., Kayama, H., Nagamune, K., Takashima, S., Matsuura, Y., Soldati-Favre, D., Takeda, K.: ATF6 $\beta$ is a host cellular target of the Toxoplasma gondii virulence factor ROP18. J Exp Med 208, 1533-1546 (2011).

44. Honore, S., Couvelard, A., Garin, Y. J., Bedel, C., Henin, D., Darde, M. L., Derouin, F.: Genotyping of Toxoplasma gondii strains from immunocompromised patients. Pathol Biol (Paris) 48, 541-547 (2000).

45. de-la-Torre, A., Sauer, A., Pfaff, A. W., Bourcier, T., Brunet, J., Speeg-Schatz, C., Ballonzoli, L., Villard, O., Ajzenberg, D., Sundar, N., Grigg, M. E., Gomez-Marin, J. E., Candolfi, E.: Severe South American ocular toxoplasmosis is associated with decreased Ifn- $\gamma / \mathrm{Il}-17 \mathrm{a}$ and increased Il-6/Il-13 intraocular levels. PLoS Negl Trop Dis 7, e2541 (2013).

46. Vallochi, A. L., Muccioli, C., Martins, M. C., Silveira, C., Belfort, R., Jr., Rizzo, L. V.: The genotype of Toxoplasma gondii strains causing ocular toxoplasmosis in humans in Brazil. Am J Ophthalmol 139, 350-351 (2005).

47. Derouin, F., Garin, Y. J.: Toxoplasma gondii: Blood and tissue kinetics during acute and chronic infections in mice. Exp Parasitol 73, 460-468 (1991).

48. Flegr, J., Zitkova, S., Kodym, P., Frynta, D.: Induction of changes in human behaviour by the parasitic protozoan Toxoplasma gondii. Parasitology 113, 49-54 (1996). 